\title{
Can liquid biopsy-guided EGFR-targeted therapy be a surrogate for the tissue-based standard approach?
}

\author{
In-Jae $\mathbf{O h}^{1,2}$ \\ ${ }^{1}$ Department of Internal Medicine, Chonnam National University Medical School, Gwangju, Republic of Korea; ${ }^{2}$ Lung and Esophageal Cancer \\ Clinic, Chonnam National University Hwasun Hospital, Jeonnam, Republic of Korea \\ Correspondence to: In-Jae Oh, MD, PhD. Lung and Esophageal Cancer Clinic, Chonnam National University Hwasun Hospital, 322 Seoyang-ro, \\ Hwasun, Jeonnam 58128, Republic of Korea. Email: droij@chonnam.ac.kr. \\ Provenance: This is an invited article commissioned by the Section Editor Hengrui Liang (Department of Thoracic Surgery, Guangzhou Medical \\ University, Guangzhou, China). \\ Comment on: Wang Z, Cheng Y, An T, et al. Detection of EGFR mutations in plasma circulating tumour DNA as a selection criterion for first-line \\ gefitinib treatment in patients with advanced lung adenocarcinoma (BENEFIT): a phase 2, single-arm, multicentre clinical trial. Lancet Respir Med \\ 2018;6:681-90.
}

Submitted May 23, 2019. Accepted for publication Jun 10, 2019.

doi: $10.21037 /$ tlcr.2019.06.03

View this article at: http://dx.doi.org/10.21037/tlcr.2019.06.03

The epidermal growth factor receptor $(E G F R)$ gene mutation has changed the clinical practice of stage IV nonsmall cell lung cancer (NSCLC) (1). Many well designed randomized trials have shown that EGFR-tyrosine kinase inhibitors (TKIs) are better treatment than conventional chemotherapy in patients who had sensitizing EGFR mutations (2,3). Therefore, identification of EGFRsensitizing mutations in tumor tissue is the current standard biomarker to identify candidates who will benefit from firstline EGFR-TKI targeted therapy (4). However, multiple factors make it difficult to obtain enough tumor tissue for $E G F R$ genetic analysis $(5,6)$. When only a small tumor specimen is available, it is more feasible to assess tumor genomics via a blood sample or other body fluid, termed a 'liquid biopsy' (7).

Although liquid biopsy most often refers to the analysis of circulating tumor DNA (ctDNA) from peripheral blood, this term also encompasses the isolation and analysis of tumor-derived material (e.g., DNA, RNA, or even intact cells) from blood or other body fluids (8). Fragments of DNA from tumor cells that are shed into the blood can be detected as ctDNA (8-14). With appropriate processing, ctDNA can be tumor specific and provide valuable molecular information through fragmented DNAs (10). Liquid biopsy is a minimally invasive, easily repeatable method that may predict the development of drug resistance by serial monitoring earlier than radiologic progression or the appearance of clinical symptoms. Therefore, liquid biopsy is becoming a valuable tool for molecular analysis, gaining new insights into tumor heterogeneity, early cancer diagnosis and monitoring for recurrence or drug resistance (8). The consensus criteria used to select firstline treatment in NSCLC patients for molecular testing of ctDNA are the same used for molecular analysis with DNA isolated from tissue $(12,15)$.

Among various types of liquid sources, easily accessible blood-based liquid biopsy has the potential to provide valuable information for clinical application of $E G F R$ targeted treatment $(12,14)$. There has been reported several retrospective studies that showed the correlation between ctDNA-based liquid biopsy and efficacy of EGFR-TKIs (16-19). The BENEFIT study was the first prospective study to approve the efficacy of first-line gefitinib therapy with ctDNA-based EGFR mutation analysis, and it provided the evidence that ctDNA-based EGFR mutation status can be used to ascertain eligibility for EGFR targeted therapy (20). Wang et al. evaluated plasma ctDNA-based EGFR mutation detection using droplet digital PCR (ddPCR). In addition, they analyzed dynamic change of EGFR-sensitizing and $E G F R$-resistance mutation statuses in relation to clinical efficacy throughout the targeted therapy. The objective response rate (ORR, $72.1 \%$ ) and the median progression- 
free survival (PFS, 9.5 months) of the BENEFIT study were similar to those of several previous reports using tissue-based EGFR analysis (2,3). Using ddPCR, the authors obtained a satisfactory specificity of $93.9 \%$ with a relatively low sensitivity of $70.0 \%$, which may limit the use of ddPCR in routine clinical practice. Nevertheless, EGFR mutation detection by ddPCR using plasma ctDNA could be used to select patients who could be benefit from first-line gefitinib when an insufficient tumor specimen is available for tissuebased molecular analysis.

The BENEFIT study showed that ctDNA-based liquid biopsy could not only assess baseline genetic status but also monitor changes over time, unlike tissue biopsy. Several interesting findings from a clinical perspective were also described in this study (20). First, the efficacy of gefitinib in patients with a de novo T790M mutation was estimated to be $5 \%$ worse than that in patients without it (ORR: $33.3 \%$ vs. $74.1 \%$; median PFS: 5.6 vs. 9.6 months, $\mathrm{P}=0.004)$. The authors suggested that the use of first-line treatment with third-generation EGFR-TKIs could be an ideal option for these patients to cover T790M mutation. Second, the loss of EGFR mutations in ctDNA at week 8, which reflects decreased tumor burden, could have a better outcome than persistent existence (median PFS: 11.0 vs. 2.1 months, $\mathrm{P}<0.0001$ ). A higher number of patients showed a loss of the EGFR exon 19 deletion than the L858R mutation (94.1\% vs. $81.7 \%, \mathrm{P}=0.017$ ). Dynamic changes in ctDNA EGFR mutation status may predict therapeutic outcomes like the other clinical trial with erlotinib (19). Third, dynamic alterations in EGFR T790M mutations could be used to predict radiological progression. The median time difference from baseline negativity to T790M positivity was 7.6 months, and that from T790M positivity to radiological progression was 2.0 months in the BENEFIT study. Therefore, we should be aware that acquired resistance could be developed in case of T790M positivity in liquid biopsy, and other strategies such as an early switch to osimertinib or a treatment combining other drugs may be needed.

Patients enrolled in the BENEFIT study also had performed next generation sequencing (NGS) on their plasma-derived ctDNA to detect additional mutations in tumor-suppressor genes and oncogenic drivers using an ultradeep (20,000×) 168-gene panel named LungPlasma ${ }^{\mathrm{TM}}$ (20). The authors classified three genetic subgroups: patients that (I) harbored only EGFR-sensitizing mutations; (II) had concurrent EGFR-sensitizing mutations and mutations in tumor-suppressor genes; (III) had multiple alterations in oncogenic drivers (MET, ERBB2, KRAS, $B R A F, R E T$, or ROS1) besides EGFR-sensitizing mutations. Median PFS for these three subgroups was 13.2, 9.3, and 4.7 months, respectively. And the median PFS was significantly longer in patients that harbored only EGFRsensitizing mutations than other subgroups (subgroup $1 v s$. subgroup 2, $\mathrm{P}=0.002$; subgroup 1 vs. subgroup $3, \mathrm{P}=0.0003$ ). Furthermore, blood-based NGS analysis confirmed the complex genetic aberrance in about $90 \%$ of the subgroup of persistent ctDNA EGFR mutations at week 8. Therefore, liquid biopsy-based analysis of baseline driver genes and subsequent dynamic changes could help to understand treatment outcome, and suggests that alternative therapeutic strategies are needed in patients with EGFR mutations who respond poorly to EGFR-TKI monotherapy.

With the development of ctDNA detection platforms, the low sensitivity issue of liquid biopsy can be solved through standardization and optimized new techniques, such ddPCR and ultradeep NGS (7). The pre-analytic standardization of liquid biopsy should include the use of plasma over serum, avoidance of heparinized tubes, prompt centrifugation, and standardization of cell free extraction methods (21). Liquid biopsies could be developed as the new standard for early detection of acquired drug resistance, with conventional tissue biopsies recommended only in ctDNA-negative cases (7). With technical improvements, the liquid biopsy-guided targeted therapy might be a surrogate for the tissue-based standard approach in the near future. It is likely that a subset of patients with stage IV NSCLC may only require plasma ctDNA liquid biopsy, possibly with the addition of circulating tumor cell or urine ctDNA analysis, thus avoiding the need for invasive tissue biopsy (7).

In conclusion, the BENEFIT study has helped advance research in this field by attempting to elucidate how the identification of EGFR mutations in ctDNA plays a crucial role in accurately identifying patients who might benefit from TKIs therapy rather than the tissue biopsybased approach. Their dynamic measurement of EGFR mutations and profiling of co-occurring gene mutations from the baseline provide considerable support for pursuing strategies of EGFR targeted therapy to prevent the evolution of acquired resistance. ctDNA analysis has rapidly emerged as a technology with many promising clinical applications. Effective clinical integration of ctDNA analysis will require a careful understanding of the advantages and limitations of this approach to properly interpret results and guide clinical decision making. Although further studies are needed, 
ctDNA analysis harbors the potential to improve precision cancer medicine.

\section{Acknowledgments}

Funding: This manuscript was supported by a grant (HCRI 19136) from the Chonnam National University Hwasun Hospital Institute for Biomedical Science.

\section{Footnote}

Conflicts of Interest: The author has no conflicts of interest to declare.

Ethical Statement: The author is accountable for all aspects of the work in ensuring that questions related to the accuracy or integrity of any part of the work are appropriately investigated and resolved.

\section{References}

1. Sharma SV, Bell DW, Settleman J, et al. Epidermal growth factor receptor mutations in lung cancer. Nat Rev Cancer 2007;7:169-81.

2. Fukuoka $M, \mathrm{Wu}$ YL, Thongprasert $\mathrm{S}$, et al. Biomarker analyses and final overall survival results from a phase III, randomized, open-label, first-line study of gefitinib versus carboplatin/paclitaxel in clinically selected patients with advanced non-small-cell lung cancer in Asia (IPASS). J Clin Oncol 2011;29:2866-74.

3. Zhou $\mathrm{C}, \mathrm{Wu} \mathrm{YL}$, Chen G, et al. Final overall survival results from a randomised, phase III study of erlotinib versus chemotherapy as first-line treatment of EGFR mutation-positive advanced non-small-cell lung cancer (OPTIMAL, CTONG-0802). Ann Oncol 2015;26:1877-83.

4. Rosell R, Karachaliou N. Circulating tumour DNA genomics in EGFR-mutant lung adenocarcinoma. Lancet Respir Med 2018;6:649-51.

5. Malapelle U, Raez LE, Serrano MJ, et al. Detection of epidermal growth factor receptor mutations in circulating tumor DNA: reviewing BENEFIT clinical trial. J Thorac Dis 2018;10:6388-91.

6. Kim TO, Oh IJ, Kho BG, et al. Feasibility of re-biopsy and EGFR mutation analysis in patients with non-small cell lung cancer. Thorac Cancer 2018;9:856-64.

7. Goldman JW, Noor ZS, Remon J, et al. Are liquid biopsies a surrogate for tissue EGFR testing? Ann Oncol 2018;29:i38-46.

8. Corcoran RB, Chabner BA. Application of Cell-free DNA Analysis to Cancer Treatment. N Engl J Med 2018;379:1754-65.

9. Crowley E, Di Nicolantonio F, Loupakis F, et al. Liquid biopsy: monitoring cancer-genetics in the blood. Nat Rev Clin Oncol 2013;10:472-84.

10. Nanjo S, Bivona TG. Circulating tumor DNA analysis in patients with EGFR mutant lung cancer. J Thorac Dis 2018;10:S4061-4.

11. Xu C, Offin M, Paik PK, et al. Liquid biopsy guided precision therapy for lung cancers. J Thorac Dis 2018;10:S4173-5.

12. Rolfo C, Mack PC, Scagliotti GV, et al. Liquid Biopsy for Advanced Non-Small Cell Lung Cancer (NSCLC): A Statement Paper from the IASLC. J Thorac Oncol 2018;13:1248-68.

13. Neumann MHD, Bender S, Krahn T, et al. ctDNA and CTCs in Liquid Biopsy - Current Status and Where We Need to Progress. Comput Struct Biotechnol J 2018;16:190-5.

14. Merker JD, Oxnard GR, Compton C, et al. Circulating Tumor DNA Analysis in Patients With Cancer: American Society of Clinical Oncology and College of American Pathologists Joint Review. J Clin Oncol 2018;36:1631-41.

15. Wu YL, Planchard D, Lu S, et al. Pan-Asian adapted Clinical Practice Guidelines for the management of patients with metastatic non-small-cell lung cancer: a CSCO-ESMO initiative endorsed by JSMO, KSMO, MOS, SSO and TOS. Ann Oncol 2019;30:171-210.

16. Bai H, Mao L, Wang HS, et al. Epidermal growth factor receptor mutations in plasma DNA samples predict tumor response in Chinese patients with stages IIIB to IV nonsmall-cell lung cancer. J Clin Oncol 2009;27:2653-9.

17. Douillard JY, Ostoros G, Cobo M, et al. Gefitinib treatment in EGFR mutated caucasian NSCLC: circulating-free tumor DNA as a surrogate for determination of EGFR status. J Thorac Oncol 2014;9:1345-53.

18. Kimura H, Suminoe M, Kasahara K, et al. Evaluation of epidermal growth factor receptor mutation status in serum DNA as a predictor of response to gefitinib (IRESSA). Br J Cancer 2007;97:778-84.

19. Mok T, Wu YL, Lee JS, et al. Detection and Dynamic Changes of EGFR Mutations from Circulating Tumor DNA as a Predictor of Survival Outcomes in NSCLC Patients Treated with First-line Intercalated Erlotinib and Chemotherapy. Clin Cancer Res 2015;21:3196-203. 
20. Wang Z, Cheng Y, An T, et al. Detection of EGFR mutations in plasma circulating tumour DNA as a selection criterion for first-line gefitinib treatment in patients with advanced lung adenocarcinoma (BENEFIT): a phase 2, single-arm, multicentre clinical trial. Lancet Respir Med
2018;6:681-90.

21. Wan JCM, Massie C, Garcia-Corbacho J, et al. Liquid biopsies come of age: towards implementation of circulating tumour DNA. Nat Rev Cancer 2017;17:223-38.

Cite this article as: Oh IJ. Can liquid biopsy-guided EGFRtargeted therapy be a surrogate for the tissue-based standard approach? Transl Lung Cancer Res 2019;8(Suppl 4):S351-S354. doi: $10.21037 /$ tlcr.2019.06.03 